\title{
Comparative genomics of environmental Aspergillus fumigatus and the role of agricultural azoles on the emergence of resistance
}

Amelia E. Barber ${ }^{1,2}$, Jennifer Born ${ }^{4}$, Ivo Schliebner ${ }^{4}$, Antonia Lange ${ }^{1,2}$, Holger B. Deising ${ }^{4}$, and Oliver Kurzai ${ }^{1,2,3}$

${ }^{1}$ Leibniz Institute for Natural Product Research and Infection Biology - Hans Knöll Institute. Jena, Germany. ${ }^{2}$ National Reference Center for Invasive Fungal Infections. Jena, Germany. ${ }^{3}$ Institute for Hygiene and Microbiology, University of Würzburg. Würzburg, Germany. ${ }^{4}$ Institute of Agricultural and Nutritional Sciences. Martin Luther University Halle-Wittenberg. Halle (Saale), Germany

Investigating the link between agriculture and drug resistance in Aspergillus fumigatus

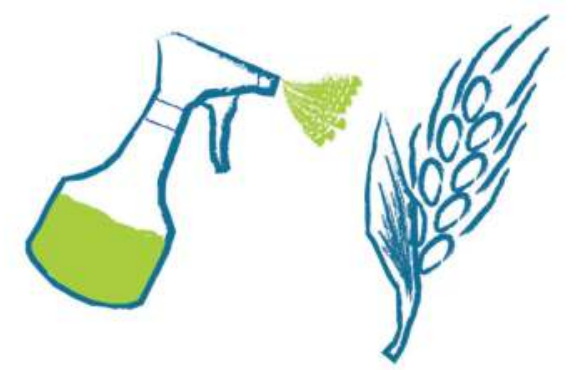

Azole Use in Agriculture

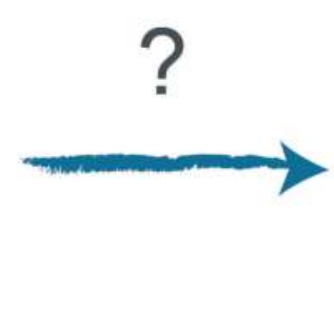

Development of Resistance
Over the last decade there has been a rapid and global emergence of azole resistance in the ubiquitous fungal pathogen Aspergillus fumigatus. The dominant resistance mechanism is of environmental origin, suggesting that resistance is emerging though a selective pressure applied by the widespread usage of azole antifungals in agriculture. However, the influence of these fungicides on the ecology of $A$. fumigatus, as well as the downstream consequences for agriculture and medicine remain largely unknown.

\section{$5 \%$ of agriculturally-isolated $A$. fumigatus} isolates show resistance to medical azoles

A. fumigatus was isolated from soil samples obtained from farms in Germany practicing organic agriculture and those practicing conventional agricultural using azole fungicides.

\begin{tabular}{|c|c|c|c|c|c|c|}
\hline & \multicolumn{3}{|c|}{ Agricultural Azoles } & \multicolumn{3}{|c|}{ Medical Azoles } \\
\hline & Tebu & Difeno I & Prothio & Itra & Vori & Posa \\
\hline \multirow{2}{*}{ Overall } & $27.3 \%$ & $88.9 \%$ & $1.9 \%$ & $6.0 \%$ & $5.1 \%$ & $1.9 \%$ \\
\hline & $59 / 216$ & $192 / 216$ & $4 / 216$ & $13 / 216$ & $11 / 216$ & $4 / 216$ \\
\hline \multirow{2}{*}{ Conventional } & $30.3 \%$ & $86.6 \%$ & $2.5 \%$ & $7.6 \%$ & $7.6 \%$ & $2.5 \%$ \\
\hline & $36 / 119$ & $103 / 119$ & 3/119 & 9/119 & 9/119 & $3 / 119$ \\
\hline \multirow{2}{*}{ Organic } & $23.7 \%$ & $91.8 \%$ & $1.0 \%$ & $4.1 \%$ & $2.1 \%$ & $1.0 \%$ \\
\hline & $23 / 97$ & $89 / 97$ & $1 / 97$ & $4 / 97$ & $2 / 97$ & $1 / 97$ \\
\hline
\end{tabular}

Isolates from conventional agriculture have a higher resistance rate than those originating from organic agriculture. Isolates resistant to medical azoles frequently possess elevated MICs against agricultural azoles, indicating cross resistance. $\approx 60 \%$ of azole-resistant $A$. fumigatus isolates do not possess known cyp51a mutations

\begin{tabular}{|c|c|c|}
\hline Itraconazole & Voriconazole & Posaconazole \\
\hline $\begin{array}{l}\mathbf{6 . 0 \%} \\
(13 / 216)\end{array}$ & $\begin{array}{l}\mathbf{5 . 1 \%} \\
(11 / 216)\end{array}$ & $\begin{array}{l}1.9 \% \\
(4 / 216)\end{array}$ \\
\hline $\begin{array}{l}\text { Genotype: } \\
8 \text { WT cyp51a } \\
4 \text { TR/L98 } \\
1 \text { Pending }\end{array}$ & $\begin{array}{l}\text { Genotype: } \\
8 \text { WT cyp51a } \\
4 \text { TR/L98 }\end{array}$ & $\frac{\text { Genotype: }}{4 \text { TR/L98 }}$ \\
\hline
\end{tabular}

Bio type ${ }^{\circledR}$

Large scale whole genome sequencing to probe the influence of azoles on the ecology of agriculturally-isolated $A$. fumigatus

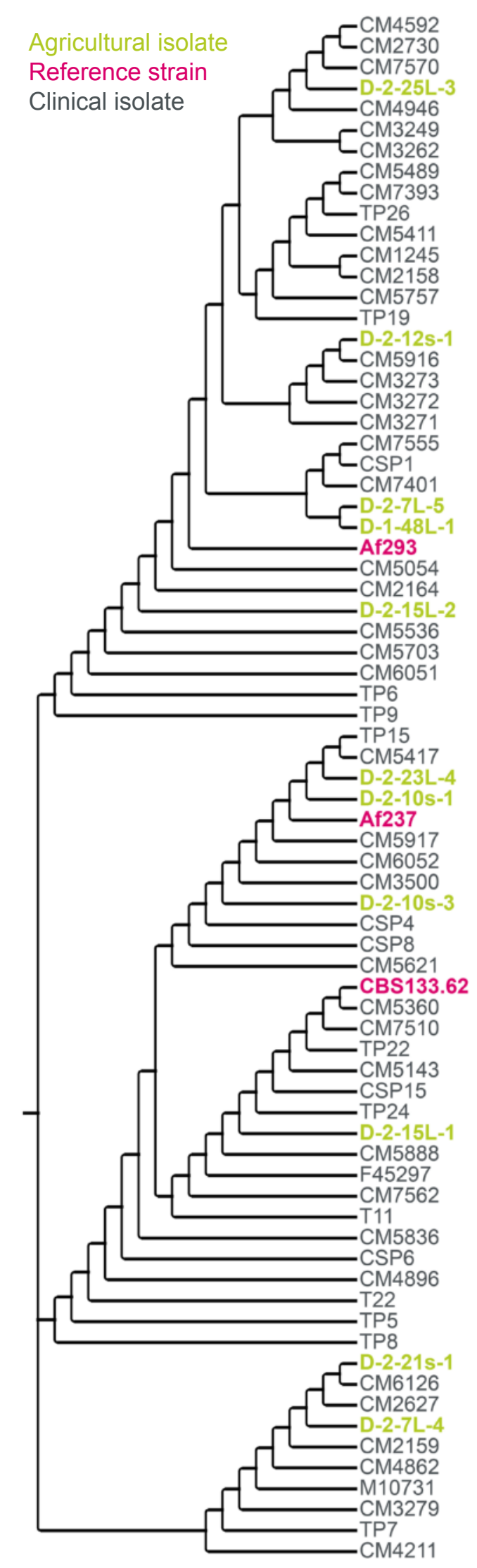

Randomly-selected isolates from the same conventional field are widely distrubted among a panel of clinical isolates. Phylogeny inferred from TRESP typing. (GarciaRubio et al. 2016).

330 isolates submitted for WGS to answer questions including:

- Is there a change in the strain population before vs. after the growing season and azole application?

- Are there differences in the populations present on conventional vs. organic fields?

- Through comparison with clinical strains, can we identify fungal determinant critical for human infection? 